\title{
Existence of positive solutions for nonlocal problems with indefinite nonlinearity
}

\section{Xiaotao Qian ${ }^{1 *}$ (D) and Wen Chao ${ }^{2}$}

\section{"Correspondence:}

qianxiaotao1984@163.com

'Department of Basic Teaching and

Research, Yango University, Fuzhou,

P.R. China

Full list of author information is

available at the end of the article

\section{Springer}

\section{Abstract}

In this paper, we consider the following new nonlocal problem:

$$
\begin{cases}-\left(a-b \int_{\Omega}|\nabla u|^{2} d x\right) \Delta u=\lambda f(x)|u|^{p-2} u, & x \in \Omega, \\ u=0, & x \in \partial \Omega,\end{cases}
$$

where $\Omega$ is a smooth bounded domain in $\mathbb{R}^{3}, a, b>0$ are constants, $3<p<6$, and the parameter $\lambda>0$. Under some assumptions on the sign-changing function $f$, we obtain the existence of positive solutions via variational methods.

MSC: $35 J 20 ; 35 J 60$

Keywords: Nonlocal problem; Variational methods; Positive solution; Indefinite nonlinearity

\section{Introduction and main resluts}

In this paper, we are concerned with the existence of positive solutions for the following new nonlocal problem:

$$
\begin{cases}-\left(a-b \int_{\Omega}|\nabla u|^{2} d x\right) \Delta u=\lambda f(x)|u|^{p-2} u, & x \in \Omega, \\ u=0, & x \in \partial \Omega,\end{cases}
$$

where $\Omega$ is a smooth bounded domain in $\mathbb{R}^{3}, a, b>0$ are constants, $3<p<6$, and the parameter $\lambda>0 . f(x)$ is sign changing in $\Omega$, which is the reason why we call it indefinite nonlinearity in the title.

In recent years, the Kirchhoff type problems in a bounded domain

$$
\begin{cases}-\left(a+b \int_{\Omega}|\nabla u|^{2} d x\right) \Delta u=f(x, u), & x \in \Omega, \\ u=0, & x \in \partial \Omega,\end{cases}
$$

have been investigated by a lot of scholars under different assumptions on $f(x, u)$, see e.g. [1-9]. There are also many studies for problem (1.2) on the whole space $\mathbb{R}^{N}(N \geq 3)$. For this case, we refer the interested readers to [10-17]. In particular, Chen [10] obtained the

(c) The Author(s) 2020. This article is licensed under a Creative Commons Attribution 4.0 International License, which permits use, sharing, adaptation, distribution and reproduction in any medium or format, as long as you give appropriate credit to the original author(s) and the source, provide a link to the Creative Commons licence, and indicate if changes were made. The images or other third party material in this article are included in the article's Creative Commons licence, unless indicated otherwise in a credit line to the material. If material is not included in the article's Creative Commons licence and your intended use is not permitted by statutory regulation or exceeds the permitted use, you will need to obtain permission directly from the copyright holder. To view a copy of this licence, visit http://creativecommons.org/licenses/by/4.0/. 
existence and multiplicity of positive solutions to the Kirchhoff problem with indefinite nonlinearity by using mountain pass theorem and minimization argument.

In problem (1.2), if we replace $a+b \int_{\Omega}|\nabla u|^{2} d x$ by $a-b \int_{\Omega}|\nabla u|^{2} d x$, it turns to be the following new nonlocal problem:

$$
\begin{cases}-\left(a-b \int_{\Omega}|\nabla u|^{2} d x\right) \Delta u=f(x, u), & x \in \Omega, \\ u=0, & x \in \partial \Omega .\end{cases}
$$

So far, there are only a few works about such a nonlocal problem. Yin and Liu [18] first studied this kind of nonlocal one and obtained two nontrivial solutions for problem (1.3) when $f(x, u)=|u|^{p-2} u$ with $2<p<2^{*}\left(2^{*}=\frac{2 N}{N-2}\right.$ when $N \geq 3$ and $2^{*}=+\infty$ when $\left.N=1,2\right)$. Lei et al. [19] proved that problem (1.3) has at least two positive solutions in the case of $f(x, u)=f_{\lambda}(x)|u|^{q-2} u$, where $1<q<2$ and $f_{\lambda}(x) \in L^{\infty}(\Omega)$ changes sign. Generalization of the result of [18] and [19] was done by Duan et al. [20]. In [20], the authors proved the existence and multiplicity results for problem (1.3) in the case of $f(x, u)=f(x)|u|^{p-2} u$, where $1 \leq p<2^{*}$ and $f(x) \in L^{\frac{2^{*}}{2^{*}-p}}(\Omega) \backslash\{0\}$ is nonnegative. Subsequently, Lei et al. [21] also obtained the multiplicity of nontrivial solutions for problem (1.3) with singularity. On the whole space $\mathbb{R}^{4}$, Wang et al. [22] researched problem (1.3) when $f(x, u)=|u|^{2} u+\mu f(x)$. Under the assumption $f(x) \in L^{4 / 3}\left(\mathbb{R}^{4}\right)$, they showed the existence of multiple positive solutions for the considered problem.

From the works described above, we do not see any existence result of positive solution to problem (1.1) in the case that $3<p<6$, the nonlocal term $a-b \int_{\Omega}|\nabla u|^{2} d x$, and indefinite nonlinearity. Thus, it is natural to ask what the case would be. Our purpose of this paper is to show some existence of positive solutions for problem (1.1).

In order to state our main results, we first make the following hypotheses on $f$ :

$\left(f_{1}\right) f(x) \in L^{\infty}(\Omega)$;

$\left(f_{2}\right)\left|\Sigma_{0}\right|=0$ and the set $\Sigma_{+}$has an interior point, where $\Sigma_{0}=\{x \in \Omega: f(x)=0\}, \Sigma_{+}=$ $\{x \in \Omega: f(x)>0\}$, and $\Sigma_{-}=\{x \in \Omega: f(x)<0\} ;$

$\left(f_{1}^{\prime}\right) f(x) \in L^{p_{*}}(\Omega)$, where $p_{*}=\frac{6}{6-p}$;

$\left(f_{2}^{\prime}\right)$ there is $d_{0}>0$ such that the set $\Sigma_{d_{0}}=\left\{x \in \Omega: f(x) \geq d_{0}\right\}$ has an interior point.

Our main results can be stated as follows.

Theorem 1.1 Assume that hypotheses $\left(f_{1}\right),\left(f_{2}\right)$ hold and $4 \leq p<6$. Then, for any $\lambda>0$, problem (1.1) has at least a positive solution.

Theorem 1.2 Assume that hypotheses $\left(f_{1}^{\prime}\right),\left(f_{2}^{\prime}\right)$ hold and $3<p<4$. Then there exists $\lambda_{*}>0$ such that, for any $\lambda \in\left(0, \lambda_{*}\right)$, problem (1.1) has at least a positive solution.

Problem (1.1) is variational in nature. Note that, for any $u \in H_{0}^{1}(\Omega)$, the functional

$$
I(u)=\frac{a}{2}\|u\|^{2}-\frac{b}{4}\|u\|^{4}-\frac{\lambda}{p} \int_{\Omega} f(x)|u|^{p} d x
$$

is well defined, where $\|u\|^{2}=\int_{\Omega}|\nabla u|^{2} d x$. Furthermore, it belongs to $C^{1}\left(H_{0}^{1}(\Omega), \mathbb{R}\right)$ and its critical points are precisely the weak solutions of problem (1.1). Here, we say $u \in H_{0}^{1}(\Omega)$ is a weak solution to problem (1.1) if, for any $v \in H_{0}^{1}(\Omega)$, it holds

$$
\left(a-b \int_{\Omega}|\nabla u|^{2} d x\right) \int_{\Omega} \nabla u \nabla v d x-\lambda \int_{\Omega} f(x)|u|^{p-2} u v d x=0 .
$$


In the proof of Theorem 1.1, the main difficulty is to prove the boundedness of the Palais-Smale ( $(P S)$ for short) sequence, which cannot be proved directly in the case of $4 \leq p<6$ and indefinite nonlinearity. We will prove the boundedness of (PS) sequence indirectly, which is inspired by [10]. In the proof of Theorem 1.2, since $\left(f_{2}^{\prime}\right)$ without assuming $\left|\Sigma_{0}\right|=0$, we cannot get the compactness property for the corresponding energy functional as in the proof of Theorem 1.1. We overcome this difficulty by pulling the energy level down below some critical level. However, we now face the situation with superlinear and indefinite nonlinearity, it is difficult to achieve the critical energy level. To this end, we choose a suitable test function presented in [23] (see also [12]). At this point, we need to require the restrictive condition $p>3$. Without this condition, we do not know if the result is still right.

Throughout this paper, we make use of the following notations. $H_{0}^{1}(\Omega)$ and $L^{q}(\Omega)$ are standard Sobolev spaces with the usual norm $\|u\|^{2}=\int_{\Omega}|\nabla u|^{2} d x,|u|_{q}^{q}=\int_{\Omega}|u|^{q} d x . f_{+}=$ $\max \{f, 0\}$ and $f_{-}=\min \{f, 0\} . B_{r}(x)$ denotes an open ball centered at $x$ with radius $r>0$. $\rightarrow$ and $\rightarrow$ denote strong and weak convergence, respectively. $C$ and $C_{i}$ denote various positive constants whose values may vary from line to line. $o(1)$ denotes a quantity such that $o(1) \rightarrow 0$ as $n \rightarrow+\infty$. All limitations hold as $n \rightarrow \infty$ unless otherwise stated. Let $S$ be the best Sobolev constant for the embedding of $H_{0}^{1}(\Omega)$ into $L^{6}(\Omega)$, that is,

$$
S=\inf _{u \in H_{0}^{1}(\Omega) \backslash\{0\}} \frac{\int_{\Omega}|\nabla u|^{2} d x}{\left(\int_{\Omega}|u|^{6} d x\right)^{1 / 3}}
$$

The paper contains two more sections, one is devoted to the proof of Theorem 1.1, the other is dedicated to the proof of Theorem 1.2.

\section{Proof of Theorem 1.1}

Lemma 2.1 Under the assumptions of Theorem 1.1, for any $\lambda>0$, there exists a sequence $\left\{u_{n}\right\} \subset H_{0}^{1}(\Omega)$ such that $I\left(u_{n}\right) \rightarrow c_{*}<\frac{a^{2}}{4 b}$ and $I^{\prime}\left(u_{n}\right) \rightarrow 0$.

Proof By $\left(f_{1}\right),(1.4)$, and Hölder's inequality, one has

$$
\begin{aligned}
I(u) & =\frac{a}{2}\|u\|^{2}-\frac{b}{4}\|u\|^{4}-\frac{\lambda}{p} \int_{\Omega} f(x)|u|^{p} d x \\
& \geq \frac{a}{2}\|u\|^{2}-\frac{b}{4}\|u\|^{4}-\frac{\lambda}{p}|f|_{\infty}\left(\int_{\Omega} 1 d x\right)^{\frac{6-p}{6}}\left(\int_{\Omega}|u|^{p \cdot \frac{6}{p}} d x\right)^{\frac{p}{6}} \\
& \geq \frac{a}{2}\|u\|^{2}-\frac{b}{4}\|u\|^{4}-\frac{\lambda}{p}|f|_{\infty}|\Omega|^{\frac{6-p}{6}} S^{-p / 2}\|u\|^{p} .
\end{aligned}
$$

Thanks to $4 \leq p<6$, the above inequality implies that, for any $\lambda>0$, there are $\alpha, \delta>0$ such that $I(u) \geq \alpha$ for all $\|u\|=\delta$.

Moreover, by $\left(f_{2}\right)$, we know that there is $u \in H_{0}^{1}(\Omega)$ satisfying $\int_{\Omega} f(x)|u|^{p} d x>0$. Then we have that

$$
\lim _{t \rightarrow+\infty} I(t u)=\lim _{t \rightarrow+\infty}\left[\frac{a}{2} t^{2}\|u\|^{2}-\frac{b}{4} t^{4}\|u\|^{4}-\frac{\lambda}{p} t^{p} \int f(x)|u|^{p} d x\right]=-\infty
$$

which means that there is $t_{0}$ large enough such that $\|e\|>\rho$ and $I(e)<0$, where $e=t_{0} u$. 
Thus, we can use the mountain pass theorem without (PS) condition [24] to obtain a sequence $\left\{u_{n}\right\} \subset H_{0}^{1}(\Omega)$ satisfying $I\left(u_{n}\right) \rightarrow c_{*}$ and $I^{\prime}\left(u_{n}\right) \rightarrow 0$ for

$$
c_{*}:=\inf _{\gamma \in \Gamma} \max _{t \in[0,1]} I(\gamma(t)) \geq \alpha>0
$$

where

$$
\Gamma:=\left\{\gamma \in C\left([0,1], H_{0}^{1}(\Omega)\right): \gamma(0)=0 \text { and } \gamma(1)=e\right\}
$$

To complete the proof of the lemma, it suffices to prove that $c_{*}<\frac{a^{2}}{4 b}$. By easy calculations, one has

$$
\begin{aligned}
\max _{t \geq 0} I(t e) & =\max _{t \geq 0}\left\{\frac{a}{2} t^{2}\|e\|^{2}-\frac{b}{4} t^{4}\|e\|^{4}-\frac{\lambda}{p} t^{p} \int_{\Omega} f(x)|e|^{p} d x\right\} \\
& <\max _{t \geq 0}\left\{\frac{a}{2} t^{2}\|e\|^{2}-\frac{b}{4} t^{4}\|e\|^{4}\right\} \\
& \leq \frac{a^{2}}{4 b} .
\end{aligned}
$$

This together with the definition of $c_{*}$ implies $c_{*}<\frac{a^{2}}{4 b}$.

Lemma 2.2 Under the assumptions of Theorem 1.1, the functional I satisfies the $(P S)_{c}$ condition with $c<\frac{a^{2}}{4 b}$.

Proof Let $\left\{u_{n}\right\} \subset H_{0}^{1}(\Omega)$ be a $(P S)_{c}$ sequence for $I$ with $c<\frac{a^{2}}{4 b}$, that is,

$$
I\left(u_{n}\right) \rightarrow c \text { and } \quad I^{\prime}\left(u_{n}\right) \rightarrow 0
$$

By (2.1), we have that

$$
c+o(1)=I\left(u_{n}\right)=\frac{a}{2} \int_{\Omega}\left|\nabla u_{n}\right|^{2} d x-\frac{b}{4}\left(\int_{\Omega}\left|\nabla u_{n}\right|^{2} d x\right)^{2}-\frac{\lambda}{p} \int_{\Omega} f(x)\left|u_{n}\right|^{p} d x,
$$

and for any $\psi \in H_{0}^{1}(\Omega)$,

$$
\begin{aligned}
o(1)\|\psi\|= & \left\langle I^{\prime}\left(u_{n}\right), \psi\right\rangle \\
= & a \int_{\Omega} \nabla u_{n} \nabla \psi d x-b\left(\int_{\Omega}\left|\nabla u_{n}\right|^{2} d x\right) \int_{\Omega} \nabla u_{n} \nabla \psi d x \\
& -\lambda \int_{\Omega} f(x)\left|u_{n}\right|^{p-2} u_{n} \psi d x .
\end{aligned}
$$

First, we show that $\left\{u_{n}\right\}$ is bounded in $H_{0}^{1}(\Omega)$. Inspired by [10], arguing by contradiction, we define $t_{n}=\left\|u_{n}\right\|$ and assume that $t_{n} \rightarrow+\infty$. Set $v_{n}=u_{n} / t_{n}$. Then we have

$$
\left\|v_{n}\right\|=\frac{\left\|u_{n}\right\|}{t_{n}}=1 \quad \text { for each } n \in \mathbb{N}
$$


Taking $\psi=u_{n}$ in (2.3), then dividing (2.2) and (2.3) by $\left\|u_{n}\right\|^{2}$, we obtain

$$
o(1)=\frac{c+o(1)}{\left\|u_{n}\right\|^{2}}=\frac{a}{2}-\frac{b}{4} \int_{\Omega}\left|\nabla u_{n}\right|^{2} d x \int_{\Omega}\left|\nabla v_{n}\right|^{2} d x-\frac{\lambda}{p} \int_{\Omega} f(x)\left|u_{n}\right|^{p-2} v_{n}^{2} d x
$$

and

$$
o(1)=\frac{o(1)\left\|u_{n}\right\|}{\left\|u_{n}\right\|^{2}}=a-b \int_{\Omega}\left|\nabla u_{n}\right|^{2} d x \int_{\Omega}\left|\nabla v_{n}\right|^{2} d x-\lambda \int_{\Omega} f(x)\left|u_{n}\right|^{p-2} v_{n}^{2} d x
$$

We distinguish two cases. In case $p=4$, multiplying (2.5) by 4 and combining with (2.6), we obtain

$$
\lim _{n \rightarrow \infty} a=0
$$

which is impossible, since $a>0$ is constant. In case $4<p<6$, we also obtain

$$
\lim _{n \rightarrow \infty} \int_{\Omega} f(x)\left|u_{n}\right|^{p-2} v_{n}^{2} d x=\frac{a p}{\lambda(4-p)}
$$

Combining this with (2.6) and $\left\|u_{n}\right\| \rightarrow \infty$, we must have

$$
\lim _{n \rightarrow \infty} \int_{\Omega}\left|\nabla v_{n}\right|^{2} d x=0
$$

which is a contradiction to (2.4).

In conclusion, either of the two cases above will lead to a contradiction. Thus, we obtain that $\left\{u_{n}\right\}$ is bounded in $H_{0}^{1}(\Omega)$. Then, up to a subsequence (still denoted by $\left\{u_{n}\right\}$ ), we may assume that

$$
\begin{cases}u_{n} \rightarrow u, & \text { in } H_{0}^{1}(\Omega), \\ u_{n} \rightarrow u, & \text { in } L^{r}(\Omega), 1 \leq r<6 \\ u_{n} \rightarrow u, & \text { a.e. in } \Omega\end{cases}
$$

By using Hölder's inequality, we obtain from (2.7) that

$$
\left.\left.\left|\int_{\Omega} f(x)\right| u_{n}\right|^{p-2} u_{n}\left(u_{n}-u\right) d x|\leq| f\right|_{\infty}\left|u_{n}\right|_{p}^{p-1}\left|u_{n}-u\right|_{p} \rightarrow 0 .
$$

It then follows from (2.1) that

$$
o(1)=\left\langle I^{\prime}\left(u_{n}\right),\left(u_{n}-u\right)\right\rangle=\left(a-b\left\|u_{n}\right\|^{2}\right) \int_{\Omega} \nabla u_{n} \nabla\left(u_{n}-u\right)+o(1),
$$

that is,

$$
a-b\left\|u_{n}\right\|^{2} \rightarrow 0 \quad \text { or } \quad \int_{\Omega} \nabla u_{n} \nabla\left(u_{n}-u\right) \rightarrow 0 .
$$

Next, we show that the former alternative above does not occur. If, to the contrary, namely,

$$
\left\|u_{n}\right\|^{2} \rightarrow \frac{a}{b}
$$


Let us define a functional by

$$
\phi(w)=\frac{1}{p} \int_{\Omega} f(x)|w|^{p} d x, \quad w \in H_{0}^{1}(\Omega) .
$$

Consequently,

$$
\left\langle\phi^{\prime}(w), v\right\rangle=\int_{\Omega} f(x)|w|^{p-2} w v d x, \quad \forall v \in H_{0}^{1}(\Omega)
$$

By using Hölder's inequality again, we obtain

$$
\begin{aligned}
& \left|\int_{\Omega} f(x)\left(\left|u_{n}\right|^{p-2} u_{n}-|u|^{p-2} u\right) v d x\right| \\
& \quad \leq\left.|f|_{\infty} \int_{\Omega}|| u_{n}\right|^{p-2} u_{n}-|u|^{p-2} u|| v \mid d x \\
& \quad \leq|f|_{\infty}\left(\left.\int_{\Omega}|| u_{n}\right|^{p-2} u_{n}-\left.|u|^{p-2} u\right|^{\frac{p}{p-1}} d x\right)^{\frac{p-1}{p}}\left(\int_{\Omega}|v|^{p} d x\right)^{\frac{1}{p}} \\
& \quad \leq\left.|f|_{\infty}|| u_{n}\right|^{p-2} u_{n}-\left.|u|^{p-2} u\right|_{\frac{p}{p-1}}|\Omega|^{\frac{6-p}{6}} S^{-p / 2}\|v\|^{p} .
\end{aligned}
$$

Hence, by using (2.7), we obtain

$$
\left\|\phi^{\prime}\left(u_{n}\right)-\phi^{\prime}(u)\right\| \leq\left.|f|_{\infty}|| u_{n}\right|^{p-2} u_{n}-\left.|u|^{p-2} u\right|_{\frac{p}{p-1}}|\Omega|^{\frac{6-p}{6}} S^{-p / 2} \rightarrow 0 .
$$

Moreover, we also have

$$
o(1)=\left\langle I^{\prime}\left(u_{n}\right), v\right\rangle=\left(a-b\left\|u_{n}\right\|^{2}\right) \int_{\Omega} \nabla u_{n} \nabla v d x-\lambda\left\langle\phi^{\prime}\left(u_{n}\right), v\right\rangle, \quad \forall v \in H_{0}^{1}(\Omega) .
$$

This with (2.9) implies that $\phi^{\prime}\left(u_{n}\right) \rightarrow 0$. Thus, we deduce from (2.10) that

$$
\left\langle\phi^{\prime}(u), v\right\rangle=\int_{\Omega} f(x)|u|^{p-2} u v d x=0, \quad \forall v \in H_{0}^{1}(\Omega) .
$$

Then we can apply the variational method fundamental lemma [25] to obtain

$$
f(x)|u(x)|^{p-2} u(x)=0, \quad \text { a.e. } x \in \Omega .
$$

Combining this with assumption $\left(f_{2}\right)$ that $\left|\Sigma_{0}\right|=0$, we obtain $u=0$.

By $\left(f_{1}\right)$ and (2.7), we have

$$
\lim _{n \rightarrow \infty} \int_{\Omega} f(x)\left|u_{n}\right|^{p} d x=\int_{\Omega} f(x)|u|^{p} d x,
$$

and hence,

$$
\phi\left(u_{n}\right)=\frac{1}{p} \int_{\Omega} f(x)\left|u_{n}\right|^{p} d x \rightarrow \frac{1}{p} \int_{\Omega} f(x)|u|^{p} d x=0 .
$$


This together with (2.9) yields

$$
I\left(u_{n}\right)=\frac{a}{2}\left\|u_{n}\right\|^{2}-\frac{b}{4}\left\|u_{n}\right\|^{4}-\frac{\lambda}{p} \int_{\Omega} f(x)\left|u_{n}\right|^{p} d x \rightarrow \frac{a^{2}}{4 b},
$$

which is a contradiction to $I\left(u_{n}\right) \rightarrow c<\frac{a^{2}}{4 b}$. Therefore, we obtain $\int_{\Omega} \nabla u_{n} \nabla\left(u_{n}-u\right) d x \rightarrow 0$, which gives $\left\|u_{n}\right\| \rightarrow\|u\|$. This and the weak convergence of $\left\{u_{n}\right\}$ in $H_{0}^{1}(\Omega)$ imply that $u_{n} \rightarrow u$ in $H_{0}^{1}(\Omega)$. The proof of Lemma 2.2 is complete.

Now, we are in a position to prove Theorem 1.1.

Proof of Theorem 1.1 In view of Lemma 2.1, there is a sequence $\left\{u_{n}\right\} \subset H_{0}^{1}(\Omega)$ such that $I\left(u_{n}\right) \rightarrow c_{*}<\frac{a^{2}}{4 b}$ and $I^{\prime}\left(u_{n}\right) \rightarrow 0$ for any $\lambda>0$. It follows from Lemma 2.2 that, along a subsequence, $u_{n} \rightarrow u$ in $H_{0}^{1}(\Omega)$, and $u$ is a weak solution of problem (1.1). Furthermore, if we replace the functional $I$ with the following one:

$$
\tilde{I}(u)=\frac{a}{2}\|u\|^{2}-\frac{b}{4}\|u\|^{4}-\frac{\lambda}{p} \int_{\Omega} f(x)\left(u_{+}\right)^{p} d x .
$$

It is clear that all the above calculations can be repeated word by word. Thus, there is a nontrivial critical point $u \in H_{0}^{1}(\Omega)$ of $\tilde{I}$. Then we have $\left\langle\tilde{I}^{\prime}(u), u_{-}\right\rangle=0$, and hence

$$
\left(a-b\|u\|^{2}\right)\left\|u_{-}\right\|^{2}=0 .
$$

Due to $u_{n} \rightarrow u$ and the fact $\left\|u_{n}\right\|^{2} \rightarrow \frac{a}{b}$ is false, we deduce $\left\|u_{-}\right\|^{2}=0$. In turn, we deduce $u \geq 0$. By the standard elliptic regularity argument and the strong maximum principle, we get $u>0$, namely, $u$ is a positive solution of problem (1.1). This finishes the proof.

\section{Proof of Theorem 1.2}

In order to prove Theorem 1.2, we need the following four lemmas.

Lemma 3.1 Suppose that $\left(f_{1}^{\prime}\right)$ holds, then the functional defined by

$$
\chi: u \in H_{0}^{1}(\Omega) \rightarrow \int_{\Omega} f(x)|u|^{p} d x
$$

is weakly continuous.

Proof It is easy to verify that the functional $\chi$ is well defined. Assume that $u_{n} \rightarrow u$ in $H_{0}^{1}(\Omega)$, then we have $u_{n} \rightarrow u$ in $L^{r}(\Omega), 2 \leq r<6$. Hence, $u_{n} \rightarrow u$ a.e. in $\Omega$. Since $\left\{u_{n}\right\}$ is bounded in $H_{0}^{1}(\Omega)$, we have $\left\{u_{n}\right\}$ is bounded in $L^{6}(\Omega)$, and so $\left\{\left|u_{n}\right|^{p}\right\}$ is bounded in $L^{6 / p}(\Omega)$. Furthermore, we obtain that

$$
\left|u_{n}\right|^{p} \rightarrow|u|^{p} \quad \text { in } L^{6 / p}(\Omega)
$$

and consequently, we can apply $\left(f_{1}^{\prime}\right)$ to deduce that

$$
\lim _{n \rightarrow+\infty} \int_{\Omega} f(x)\left|u_{n}\right|^{p} d x=\int_{\Omega} f(x)|u|^{p} d x
$$

Thus, the proof is complete. 
Lemma 3.2 Under the assumptions of Theorem 1.2, the functional I satisfies the mountainpass geometry:

(i) there exist $\rho, \beta>0$ such that $I(u) \geq \beta>0$ for all $\|u\|=\rho$;

(ii) there exists $e \in H_{0}^{1}(\Omega)$ with $\|e\|>\rho$ such that $I(e)<0$.

Proof (i) By $\left(f_{2}^{\prime}\right)$, Hölder's inequality, and (1.4), we have that

$$
\begin{aligned}
I(u) & =\frac{a}{2}\|u\|^{2}-\frac{b}{4}\|u\|^{4}-\frac{\lambda}{p} \int_{\Omega} f(x)|u|^{p} d x \\
& \geq \frac{a}{2}\|u\|^{2}-\frac{b}{4}\|u\|^{4}-\frac{\lambda}{p} \int_{\Omega} f_{+}(x)|u|^{p} d x \\
& \geq \frac{a}{2}\|u\|^{2}-\frac{b}{4}\|u\|^{4}-\frac{\lambda}{p}\left|f_{+}\right|_{p_{*}}|u|_{6}^{p} \\
& \geq \frac{a}{2}\|u\|^{2}-\frac{b}{4}\|u\|^{4}-\frac{\lambda}{p}\left|f_{+}\right|_{p_{*}} S^{-p / 2}\|u\|^{p} .
\end{aligned}
$$

As $3<p<4$, there exist $\rho, \beta>0$ such that $I(u) \geq \beta>0$ for all $\|u\|=\rho$.

(ii) The proof is similar to Lemma 2.1 .

Lemma 3.3 Suppose that the assumptions of Theorem 1.2 hold. Let $\left\{u_{n}\right\} \subset H_{0}^{1}(\Omega)$ be a $(P S)_{c}$ sequence for the functional $I$, then

(i) there exists $m>0$ such that $\left\|u_{n}\right\| \leq m$ for each $n \in \mathbb{N}$;

(ii) $\left\{u_{n}\right\}$ has a convergent subsequence if $c<\frac{a^{2}}{4 b}-D_{0} \lambda$, where $D_{0}=\left(\frac{1}{p}-\frac{1}{4}\right)\left|f_{+}\right|_{p_{*}} S^{-p / 2} m^{p}$.

Proof (i) Let $\left\{u_{n}\right\} \subset H_{0}^{1}(\Omega)$ be a $(P S)_{c}$ sequence for $I$, i.e.,

$$
\lim _{n \rightarrow+\infty} I\left(u_{n}\right)=c \text { and } \lim _{n \rightarrow+\infty} I^{\prime}\left(u_{n}\right)=0 .
$$

By (3.1), we have that, for $3<p<4$,

$$
\begin{aligned}
c+1+o(1)\left\|u_{n}\right\| & \geq I\left(u_{n}\right)-\frac{1}{p}\left\langle I^{\prime}\left(u_{n}\right), u_{n}\right\rangle \\
& =a\left(\frac{1}{2}-\frac{1}{p}\right)\left\|u_{n}\right\|^{2}-b\left(\frac{1}{4}-\frac{1}{p}\right)\left\|u_{n}\right\|^{4} \\
& \geq a\left(\frac{1}{2}-\frac{1}{p}\right)\left\|u_{n}\right\|^{2},
\end{aligned}
$$

which implies that $\left\{u_{n}\right\}$ is bounded in $H_{0}^{1}(\Omega)$, that is, there exists $m>0$ such that $\left\|u_{n}\right\| \leq m$ for each $n \in \mathbb{N}$.

(ii) After passing to a subsequence, we may assume that

$$
\begin{cases}u_{n} \rightarrow u_{*}, & \text { in } H_{0}^{1}(\Omega), \\ u_{n} \rightarrow u_{*}, & \text { in } L^{r}(\Omega), 1 \leq r<6 \\ u_{n} \rightarrow u_{*}, & \text { a.e. in } \Omega .\end{cases}
$$

First, we show that

$$
\lim _{n \rightarrow \infty} \int_{\Omega} f(x)\left|u_{n}\right|^{p-2} u_{n} v=\int_{\Omega} f(x)\left|u_{*}\right|^{p-2} u_{*} v, \quad \forall v \in H_{0}^{1}(\Omega) .
$$


To prove (3.3), let us consider $\varphi \in C_{0}^{\infty}(\Omega)$. Recalling that $p_{*}=\frac{6}{6-p}$, we can choose $r_{0}<6$ and near 6 satisfying

$$
\frac{6}{6-p}>\frac{r_{0}}{\left(r_{0}+1\right)-p}
$$

As a consequence, there is $r_{*}>1$ such that

$$
\frac{1}{p_{*}}+\frac{1}{r_{0} /(p-1)}+\frac{1}{r_{*}}=1
$$

The strong convergence $u_{n} \rightarrow u_{*}$ in $L^{r_{0}}(\Omega)$ gives $\phi_{r_{0}} \in L^{r_{0}}(\Omega)$ satisfying $\left|u_{n}(x)\right| \leq \phi_{r_{0}}(x)$ a.e. in $\Omega$. Therefore, using Young's inequality, we obtain

$$
\begin{aligned}
\left.|f(x)| u_{n}(x)\right|^{p-2} u_{n}(x) \varphi(x) \mid & \leq C\left(|f(x)|^{p_{*}}+\left|u_{n}(x)\right|^{r_{0}}+|\varphi(x)|^{r_{*}}\right) \\
& \leq C\left(|f(x)|^{p_{*}}+\left|\phi_{r_{0}}(x)\right|^{r_{0}}+|\varphi(x)|^{r_{*}}\right),
\end{aligned}
$$

a.e. in $\Omega$. Since $\varphi(x)$ is smooth, the right-hand side above belongs to $L^{1}(\Omega)$. Then we can infer from the Lebesgue theorem that

$$
\lim _{n \rightarrow \infty} \int_{\Omega} f(x)\left|u_{n}\right|^{p-2} u_{n} \varphi=\int_{\Omega} f(x)\left|u_{*}\right|^{p-2} u_{*} \varphi
$$

By density, we further obtain (3.3) holds.

Set $v_{n}=u_{n}-u_{*}$ and we claim that $\left\|v_{n}\right\| \rightarrow 0$. If not, we may suppose $\left\|v_{n}\right\| \rightarrow l$ with $l>0$. From (3.1), it follows that $\left\langle I^{\prime}\left(u_{n}\right), u_{*}\right\rangle=o(1)$. Combining this with (3.2) and (3.3), we obtain

$$
0=a\left\|u_{*}\right\|^{2}-b\left(l^{2}+\left\|u_{*}\right\|^{2}\right)\left\|u_{*}\right\|^{2}-\lambda \int_{\Omega} f(x)\left|u_{*}\right|^{p} d x
$$

Moreover, by $\left\langle I^{\prime}\left(u_{n}\right), u_{n}\right\rangle=o(1)$, we can use (3.2) and Lemma 3.1 to obtain

$$
\begin{aligned}
0= & a\left(\left\|v_{n}\right\|^{2}+\left\|u_{*}\right\|^{2}\right)-b\left(\left\|v_{n}\right\|^{4}+2\left\|v_{n}\right\|^{2}\left\|u_{*}\right\|^{2}+\left\|u_{*}\right\|^{4}\right) \\
& -\lambda \int_{\Omega} f(x)\left|u_{*}\right|^{p} d x+o(1) .
\end{aligned}
$$

Combining (3.4) and (3.5), we have

$$
o(1)=a\left\|v_{n}\right\|^{2}-b\left\|v_{n}\right\|^{4}-b\left\|v_{n}\right\|^{2}\left\|u_{*}\right\|^{2} .
$$

Passing the limit as $n \rightarrow \infty$, we get that

$$
l^{2}\left(a-b l^{2}-b\left\|u_{*}\right\|^{2}\right)=0,
$$

that is,

$$
l^{2}=\frac{a}{b}-\left\|u_{*}\right\|^{2}
$$


By (3.4) and Hölder's inequality, we obtain for $3<p<4$

$$
\begin{aligned}
I\left(u_{*}\right) & =\frac{a}{2}\left\|u_{*}\right\|^{2}-\frac{b}{4}\left\|u_{*}\right\|^{4}-\frac{\lambda}{p} \int_{\Omega} f(x)\left|u_{*}\right|^{p} d x \\
& =\frac{a}{4}\left\|u_{*}\right\|^{2}+\frac{b}{4} l^{2}\left\|u_{*}\right\|^{2}-\lambda\left(\frac{1}{p}-\frac{1}{4}\right) \int_{\Omega} f(x)\left|u_{*}\right|^{p} d x \\
& \geq \frac{a}{4}\left\|u_{*}\right\|^{2}+\frac{b}{4} l^{2}\left\|u_{*}\right\|^{2}-\lambda\left(\frac{1}{p}-\frac{1}{4}\right) \int_{\Omega} f_{+}(x)\left|u_{*}\right|^{p} d x \\
& \geq \frac{a}{4}\left\|u_{*}\right\|^{2}+\frac{b}{4} l^{2}\left\|u_{*}\right\|^{2}-\lambda\left(\frac{1}{p}-\frac{1}{4}\right)\left|f_{+}\right|_{p_{*}} S^{-p / 2}\left\|u_{*}\right\|^{p} \\
& \geq \frac{a}{4}\left\|u_{*}\right\|^{2}+\frac{b}{4} l^{2}\left\|u_{*}\right\|^{2}-\lambda\left(\frac{1}{p}-\frac{1}{4}\right)\left|f_{+}\right|_{p_{*}} S^{-p / 2} m^{p} .
\end{aligned}
$$

Furthermore, we can use (3.6)-(3.8) and Lemma 3.1 to obtain

$$
\begin{aligned}
c+o(1)= & I\left(u_{n}\right) \\
= & \frac{a}{2}\left\|u_{n}\right\|^{2}-\frac{b}{4}\left\|u_{n}\right\|^{4}-\frac{\lambda}{p} \int f(x)\left|u_{n}\right|^{p} d x \\
= & \frac{a}{2}\left\|u_{*}\right\|^{2}-\frac{b}{4}\left\|u_{*}\right\|^{4}-\frac{\lambda}{p} \int f(x)\left|u_{*}\right|^{p} d x \\
& +\frac{a}{2}\left\|v_{n}\right\|^{2}-\frac{b}{4}\left\|v_{n}\right\|^{4}-\frac{b}{2}\left\|v_{n}\right\|^{2}\left\|u_{*}\right\|^{2}+o(1) \\
= & I\left(u_{*}\right)+\frac{a}{2}\left\|v_{n}\right\|^{2}-\frac{b}{4}\left\|v_{n}\right\|^{4}-\frac{b}{2}\left\|v_{n}\right\|^{2}\left\|u_{*}\right\|^{2}+o(1) \\
= & I\left(u_{*}\right)+\frac{a}{2}\left\|v_{n}\right\|^{2}-\frac{1}{4}\left(a\left\|v_{n}\right\|^{2}-b\left\|v_{n}\right\|^{2}\left\|u_{*}\right\|^{2}\right)-\frac{b}{2}\left\|v_{n}\right\|^{2}\left\|u_{*}\right\|^{2}+o(1) \\
= & I\left(u_{*}\right)+\frac{a}{4}\left\|v_{n}\right\|^{2}-\frac{b}{4}\left\|v_{n}\right\|^{2}\left\|u_{*}\right\|^{2}+o(1) \\
= & I\left(u_{*}\right)+\frac{a}{4} l^{2}-\frac{b}{4} l^{2}\left\|u_{*}\right\|^{2}+o(1) \\
= & I\left(u_{*}\right)+\frac{a}{4}\left(\frac{a}{b}-\left\|u_{*}\right\|^{2}\right)-\frac{b}{4} l^{2}\left\|u_{*}\right\|^{2}+o(1) \\
\geq & I\left(u_{*}\right)+\frac{a^{2}}{4 b}-\frac{a}{4}\left\|u_{*}\right\|^{2}-\frac{b}{4} l^{2}\left\|u_{*}\right\|^{2}+o(1) \\
a^{2} & \lambda\left(\frac{1}{p}-\frac{1}{4}\right)\left|f_{+}\right|_{p_{*}} S^{-p / 2} m^{p} \\
& \\
&
\end{aligned}
$$

which is a contradiction with our assumption $c<\frac{a^{2}}{4 b}-D_{0} \lambda$. Thus, the claim follows, that is, $u_{n} \rightarrow u_{*}$ in $H_{0}^{1}(\Omega)$. We complete the proof of Lemma 3.3.

By condition $\left(f_{2}^{\prime}\right)$, we can choose $x_{0} \in \operatorname{int}\left(\Sigma_{d_{0}}\right)$ and $\eta>0$ small enough such that $B_{2 \eta}\left(x_{0}\right) \subset$ $\Sigma_{d_{0}}$. Define a cutoff function $\varphi(x)$ satisfying $\varphi(x) \equiv 1$ in $B_{\eta}\left(x_{0}\right), \varphi(x) \equiv 0$ outside $B_{2 \eta}\left(x_{0}\right)$ and $0 \leq \varphi \leq 1$. Inspired by [23] (see also [12]), we consider the following test function:

$$
u_{\varepsilon}(x)=\varphi(x) \frac{1}{\left(\varepsilon^{2}+\left|x-x_{0}\right|^{2}\right)^{1 / 2}}
$$

Without loss of generality, we may assume $x_{0}=0$. 
Lemma 3.4 Under the assumptions of Theorem 1.2, there exists $\lambda_{*}>0$ such that, for any $\lambda \in\left(0, \lambda_{*}\right)$,

$$
\sup _{t>0} I\left(t u_{\varepsilon}\right)<\frac{a^{2}}{4 b}-D_{0} \lambda
$$

where $D_{0}$ is defined as in Lemma 3.3.

Proof Let $\lambda_{*}=\frac{a^{2}}{4 b D_{0}}$ and note that, for any $\lambda \in\left(0, \lambda_{*}\right)$, there holds

$$
\frac{a^{2}}{4 b}-D_{0} \lambda>0
$$

It is clear that $\lim _{t \rightarrow 0^{+}} I\left(t u_{\varepsilon}\right)=0$, and so there is $t_{1}>0$ such that, for any $\lambda \in\left(0, \lambda_{*}\right)$,

$$
\sup _{0<t \leq t_{1}} I\left(t u_{\varepsilon}\right)<\frac{a^{2}}{4 b}-D_{0} \lambda
$$

In the following, we discuss the case $t>t_{1}$. Noting that $u_{\varepsilon}=0$ in $\Omega \backslash \Sigma_{d_{0}}$ and $f_{-}(x)=0$ in $\Sigma_{d_{0}}$, we have

$$
\int_{\Omega} f_{-}(x)\left|u_{\varepsilon}\right|^{p} d x=0 .
$$

Set

$$
R_{0}=\min \left\{\eta, 2^{\frac{2-p}{2(p-3)}} t_{1}^{\frac{p}{p-3}}\left(\frac{\pi d_{0}}{3 p D_{0}}\right)^{\frac{1}{p-3}}\right\}
$$

and let $\varepsilon<R_{0}$, then by $\left(f_{2}^{\prime}\right)$ we obtain

$$
\begin{aligned}
\int_{\Omega} f(x)\left|u_{\varepsilon}\right|^{p} d x & =\int_{\Sigma_{d_{0}}} f_{+}(x)\left|u_{\varepsilon}\right|^{p} d x \\
& \geq \int_{B_{R_{0}}(0)} f_{+}(x) \frac{1}{\left(\varepsilon^{2}+|x|^{2}\right)^{p / 2}} d x \\
& \geq \frac{1}{\left(2 R_{0}^{2}\right)^{p / 2}} \int_{B_{R_{0}}(0)} f_{+}(x) d x \\
& \geq \frac{1}{2^{p / 2}} \frac{4 \pi d_{0}}{3 R_{0}^{p-3}} .
\end{aligned}
$$

By (3.10), we have that, for any $\lambda \in\left(0, \lambda_{*}\right)$ and $t>t_{1}$,

$$
\begin{aligned}
I\left(t u_{\varepsilon}\right) & =\frac{a}{2} t^{2}\left\|u_{\varepsilon}\right\|^{2}-\frac{b}{4} t^{4}\left\|u_{\varepsilon}\right\|^{4}-\lambda \frac{t^{p}}{p} \int_{\Omega} f(x) u_{\varepsilon}^{p} d x \\
& \leq \sup _{t>0}\left\{\frac{a 2^{2}}{t}\left\|u_{\varepsilon}\right\|^{2}-\frac{b}{4} t^{4}\left\|u_{\varepsilon}\right\|^{4}\right\}-\lambda \frac{t_{1}^{p}}{p} \int_{\Omega} f(x) u_{\varepsilon}^{p} d x \\
& \leq \frac{a^{2}}{4 b}-\frac{t_{1}^{p}}{p} \frac{1}{2^{p / 2}} \frac{4 \pi d_{0}}{3 R_{0}^{p-3}} \lambda
\end{aligned}
$$




$$
\begin{aligned}
& \leq \frac{a^{2}}{4 b}-2 D_{0} \lambda \\
& <\frac{a^{2}}{4 b}-D_{0} \lambda .
\end{aligned}
$$

Combining (3.9) and (3.11), we have that, for any $\lambda \in\left(0, \lambda_{*}\right)$,

$$
\sup _{t>0} I\left(t u_{\varepsilon}\right)<\frac{a^{2}}{4 b}-D_{0} \lambda
$$

This completes the proof of Lemma 3.4.

Now, we are ready to prove Theorem 1.2.

Proof of Theorem 1.2 Define the minimax level of the mountain pass theorem as in Lemma 2.1. By the definition of $c_{*}$ and Lemma 3.4, we know there is $\lambda_{*}>0$ such that $c_{*}<\frac{a^{2}}{4 b}-D_{0} \lambda$ for any $\lambda \in\left(0, \lambda_{*}\right)$. According to Lemma 3.3, we further obtain the compactness property on the level $c_{*}$. Then we can argue as in the proof of Theorem 1.1 to conclude that there exists $u_{*} \in H_{0}^{1}(\Omega)$ such that $u_{*}$ is a positive solution of problem (1.1). This completes the proof.

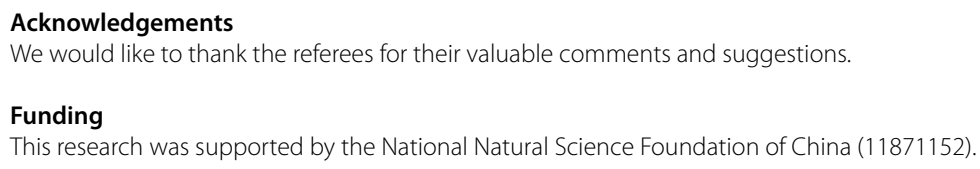

\section{Author details}

${ }^{1}$ Department of Basic Teaching and Research, Yango University, Fuzhou, P.R. China. ${ }^{2}$ School of Management, Fujian University of Technology, Fuzhou, P.R. China.

\section{Publisher's Note}

Springer Nature remains neutral with regard to jurisdictional claims in published maps and institutional affiliations.

Received: 29 October 2019 Accepted: 18 February 2020 Published online: 28 February 2020

\section{References}

1. Alves, C.O., Corrêa, F.J.S.A., Ma, T.F.: Positive solutions for a quasilinear elliptic equation of Kirchhoff type. Comput. Math. Appl. 49, 85-93 (2005)

2. Cheng, B., Wu, X.: Existence results of positive solutions of Kirchhoff type problems. Nonlinear Anal. 71, 4883-4892 (2009)

3. Duan, Y., Sun, X., Liao, J.F.: Multiplicity of positive solutions for a class of critical Sobolev exponent problems involving Kirchhoff-type nonlocal term. Comput. Math. Appl. 75, 4427-4437 (2018)

4. Fiscella, A., Valdinoci, E.: A critical Kirchhoff type problem involving a nonlocal operator. Nonlinear Anal. 94, 156-170 (2014)

5. He, X.M., Zou, W.M.: Infinitely many positive solutions for Kirchhoff-type problems. Nonlinear Anal. 70, 1407-1414 (2009)

6. Mao, A.M., Zhang, Z.T.: Sign-changing and multiple solutions of Kirchhoff type problems without the P.S. condition. Nonlinear Anal. 70, 1275-1287 (2009)

7. Naimen, D.: The critical problem of Kirchhoff type elliptic equations in dimension four. J. Differ. Equ. 257, 1168-1193 (2014) 
8. Perera, K., Zhang, Z.T.: Nontrivial solutions of Kirchhoff-type problems via Yang index. J. Differ. Equ. 221, 246-255 (2006)

9. Sun, Y.J., Liu, X.: Existence of positive solutions for Kirchhoff type problems with critical exponent. J. Partial Differ. Equ. 25, 187-198 (2012)

10. Chen, J.Q.: Multiple positive solutions to a class of Kirchhoff equation on $\mathbb{R}^{3}$ with indefinite nonlinearity. Nonlinear Anal. 96, 134-145 (2014)

11. Gan, W.B., Liu, S.B.: Multiple positive solutions of a class of Schrödinger-Poisson equation involving indefinite nonlinearity in $\mathbb{R}^{3}$. Appl. Math. Lett. 93,111-116 (2019)

12. Li, G.B., Ye, H.Y.: Existence of positive solutions for nonlinear Kirchhoff type problems in $\mathbb{R}^{3}$ with critical Sobolev exponent and sign-changing nonlinearities. Math. Methods Appl. Sci. 7, 97-114 (2013)

13. Nie, J.J.: Existence and multiplicity of nontrivial solutions for a class of Schrödinger-Kirchhoff-type equations. J. Math. Anal. Appl. 417, 65-79 (2014)

14. Qian, X.T., Chen, J.Q.: Existence of multiple solutions and estimates of extremal values for a Kirchhoff type problem with fast increasing weight and critical nonlinearity. Electron. J. Differ. Equ. 2018, Article ID 144 (2018)

15. Qian, X.T., Chao, W.: Positive solutions for a Kirchhoff type problem with fast increasing weight and critical nonlinearity. Electron. J. Qual. Theory Differ. Equ. 2019, Article ID 27 (2019)

16. Wu, X.: Existence of nontrivial solutions and high energy solutions for Schrödinger-Kirchhoff-type equations in $\mathbb{R}^{N}$. Nonlinear Anal., Real World Appl. 12, 1278-1287 (2011)

17. Xie, Q.L., Ma, S.W., Zhang, X.: Bound state solutions of Kirchhoff type problems with critical exponent. J. Differ. Equ. 261, 890-924 (2016)

18. Yin, G.S., Liu, J.S.: Existence and multiplicity of nontrivial solutions for a nonlocal problem. Bound. Value Probl. 2015, Article ID $26(2015)$

19. Lei, C.Y., Liao, J.F., Suo, H.M.: Multiple positive solutions for a class of nonlocal problems involving a sign-changing potential. Electron. J. Differ. Equ. 2017, Article ID 9 (2017)

20. Duan, Y., Sun, X., Li, H.Y.: Existence and multiplicity of positive solutions for a nonlocal problem. J. Nonlinear Sci. Appl. 10, 6056-6061 (2017)

21. Lei, C.Y., Chu, C.M., Suo, H.M.: Positive solutions for a nonlocal problem with singularity. Electron. J. Differ. Equ. 2018, Article ID 85 (2017)

22. Wang, Y., Suo, H.M., Lei, C.Y.: Multiple positive solutions for a nonlocal problem involving critical exponent. Electron. J. Differ. Equ. 2017, Article ID 275 (2017)

23. Brézis, H., Nirenberg, L.: A minimization problem with critical exponent and nonzero data in "symmetry in nature". Sc. Norm. Super. Pisa 7, 129-140 (1989)

24. Ambrosetti, A., Rabinowitz, P.H.: Dual variational methods in critical points theory and applications. J. Funct. Anal. 14 349-381 (1973)

25. Lu, W.D.: The Variational Method in Differential Equation. Sichuan University Press, Sichun (1995)

\section{Submit your manuscript to a SpringerOpen ${ }^{\circ}$ journal and benefit from:}

- Convenient online submission

- Rigorous peer review

- Open access: articles freely available online

- High visibility within the field

Retaining the copyright to your article

Submit your next manuscript at $\gg$ springeropen.com 